\title{
SYNTHESIS, CHARACTERIZATION AND EVALUATION OF BIOLOGICAL ACTIVITY OF SOME SUBSTITUTED(E)-2- BENZYLIDENEHYDRAZINECARBOTHIO AMIDES
}

\author{
R. Vijayakumar ${ }^{\mathrm{a}}$, M. Rajarajan ${ }^{\mathrm{a}}$, R.Senbagam ${ }^{\mathrm{a}}, \mathrm{S}$. Balaji ${ }^{\mathrm{a}}$, V. Manikandan ${ }^{\mathrm{a}}$, \\ G. Vanangamudia, and G. Thirunarayanan ${ }^{\mathrm{b},{ }^{*}}$
}

\author{
aPG \& Research Department of Chemistry, Government Arts College, C-Mutlur-608 102, \\ Chidambaram, India \\ ${ }^{b}$ Department of Chemistry, Annamalai University, Annamalainagar-608002,India \\ Corresponding Author: drgtnarayanan@gmail.com
}

Keywords: synthesis, substituted (E)-2-benzylidenehydrazinecarbothioamide compounds, UV, IR \& NMR spectra and Antimicrobial activities

\begin{abstract}
A series of substituted (E)-2-benzylidenehydrazinecarbothioamide compounds were synthesized by condensation of thiosemicarbazide with substituted benzaldehydes. The synthesized substituted (E)-2-benzylidenehydrazinecarbothioamide compounds were characterized by their physical constants, UV, IR and NMR spectra. The antimicrobial activities of these synthesized substituted $(E)$-2-benzylidenehydrazinecarbothioamide compounds have been screened by BauerKirby method using human pathogenic bacteria and fungal species. The antimicrobial activities of all synthesized (E)-2-benzylidenehydrazinecarbothioamide compounds have shown significant activity.
\end{abstract}

\section{INTRODUCTION}

The synthesis, structure and biological activity of some new hydrazones prepared from aliphatic hydrazides has been the focus of research. Different methods have been employed to synthesize different types of hydrazones from different starting materials. Hydrazones are characterized by the presence of the triatomic grouping $\mathrm{C}=\mathrm{N}-\mathrm{N}-$. They can be considered as Schiff bases derived from acid hydrazides. The most important property of hydrazones is their high physiological activity [1-6]. Extensive studies have revealed that the lone pair on trigonally hybridized nitrogen atom of the azomethine group is responsible [7-11] for the chemical and biological activity. It has been reported that metal complexes of hydrazones have diverse applications. They find use as plasticizers, polymerization inhibitors and antioxidants. They are used as fungicides and pesticides in biological and biochemical context.

Moreover, the hydrazone group plays an important role of the antimicrobial and possesses interesting antibacterial, antifungal [12-14] and anti-tubercular activities [15-20]. In addition, their varied coordinating behaviour makes them interesting candidates for metal-based drugs. Generally, the ligands act synergistically with metals towards their biological activity. These observations have guided the development of new hydrazones with varied biological activities [21].The biological activity of complexes derived from hydrazones have been studied and contrasted with regard to their antibacterial, antitumoral, antiviral, antimalarial and antitubercular properties [22]. It has also been shown that the azomethine $\mathrm{N}$, which has a lone pair of electrons in a sp ${ }^{2}$ hybridised orbital, is biologically important [23].

Hydrazones constitute an important class of biologically active drug molecules [24] which has attracted attention of medicinal chemists due to their wide range of pharmacological properties. These compounds are being synthesized as drugs by many researchers in order to combat diseases with minimal toxicity and maximal effects. These predictions has provided therapeutic pathway to develop new effective biologically active hydrazones. A number of hydrazone derivatives have been reported to exert notably antimicrobial, antihypertensive, anticonvulsant, analgesic, anti- 
inflammatory, antituberculosis, antitumoral, antiproliferative and antimalarial activities, biological activities of various hydrazones are well reported in literature. This review highlights diverse pharmacological activities shown by hydrazones.

Medicinal chemists have also carried out considerable research for novel antimicrobial and anticancer agents bearing hydrazone moiety. Some studies have confirmed that hydrazone derivatives exhibit antifungal and anticancer activities [25-29]. Some researchers have reported anticancer effects of some antifungal agents and carried out considerable research for deciphering the underlying mechanisms of antitumor activity [30-32].In antifungal and anticancer drug design, the lack of selectivity of conventional chemotherapeutic agents and the acquisition of multiple-drug resistance are two major challenging problems. As a consequence of this situation, the search for new effective chemotherapeutic agents has attracted a great deal of interest [33-35].

Several hydrazone derivatives have been reported as insecticides, nematocides, herbicides, rodenticides and antituburculosis in addition to that some of the hydrazone were found to be active against leukemia, sarcoma and illnesses [36,37]. we now carry out another systematic study of their synthesis and biological activity. Herein, the synthesis of the substituted (E)-2benzylidenehydrazinecarbothioamides are described and their antimicrobial properties are evaluated

\section{EXPERIMENTAL}

\subsection{General}

All the chemicals used in the present investigation, have been procured from Sigma-Aldrich Chemical Company. Melting points of all substituted $(E)$-2-benzylidenehydrazinecarbothioamides have been determined in open glass capillaries on a Mettler FP51 melting point apparatus and are uncorrected. The UV spectra of all the substituted $(E)$-2-benzylidenehydrazinecarbothioamides have been recorded with ELICO-BL222 spectrophotometer $\left(\lambda_{\max } \mathrm{nm}\right)$ in spectral grade methanol solvent. Infrared spectra $\left(\mathrm{KBr}, 4000-400 \mathrm{~cm}^{-1}\right)$ have been recorded on SHIMADZU Fourier transform spectrophotometer. Bruker AV400 NMR spectrometer operating at $400 \mathrm{MHz}$ has been utilized for recording ${ }^{1} \mathrm{H}$ NMR spectra and $100 \mathrm{MHz}$ for ${ }^{13} \mathrm{C}$ NMR spectra in DMSO solvent using TMS as internal standard.

\section{2. Synthesis of substituted $(E)$-2-benzylidenehydrazinecarbothio amides}

A solution of equimolar quantities of thiosemicarbazide $(0.01 \mathrm{~mol})$ with substituted benzaldehydes $(0.01 \mathrm{~mol})$ acetic acid (two drops) and $10 \mathrm{ml}$ of ethanol were shaken occasionally for 1 hour [38]. The completion of the reaction was monitored by TLC continuously. The resultant mixture was cooled at room temperature. Then the precipitate obtained, was filtered at the filter pump and washed several times with cold water then pale yellow solid was obtained as the final product. This crude product was recrystallized from ethanol and glittering colorless solid was obtained. The general scheme for preparation of substituted (E)-2benzylidenehydrazinecarbothioamides has shown in scheme-1.<smiles>[X]c1ccccc1/C=N/NC(N)=S</smiles>

$$
\mathrm{X}=\mathrm{H}, 3-\mathrm{Br}, 4-\mathrm{Br}, 3-\mathrm{Cl}, 4-\mathrm{Cl}, 4-\mathrm{F}, 4-\mathrm{OCH}_{3}, 4-\mathrm{CH}_{3}, 3-\mathrm{NO}_{2}, 4-\mathrm{NO}_{2}
$$

Scheme 1. Synthesis of substituted (E)-2-benzylidenehydrazinecarbothioamides

The physical constants of substituted $(E)$-2-benzylidenehydrazinecarbothioamide compounds presented in Table1. The ultraviolet absorption maxima $\left(\lambda_{\max }, \mathrm{nm}\right)$, infrared absorptions $\left(v, \mathrm{~cm}^{-1}\right)$ and NMR chemical shifts $(\delta, \mathrm{ppm})$ of substituted $(E)$-2-benzylidenehydrazinecarbothioamides are presented in Table 2. 
Table 1.Physical constants of substituted $(E)$-2-benzylidenehydrazinecarbothioamides

\begin{tabular}{|c|c|c|c|c|c|}
\hline Entry & $\mathrm{X}$ & $\mathrm{M} . \mathrm{F}$. & $\mathrm{M} . \mathrm{W}$ & Yield (\%) & m.p. (C) \\
\hline 1 & $\mathrm{H}$ & $\mathrm{C}_{8} \mathrm{H}_{9} \mathrm{~N}_{3} \mathrm{~S}$ & 179.24 & 89 & $157-158$ \\
\hline 2 & $3-\mathrm{Br}$ & $\mathrm{C}_{8} \mathrm{H}_{8} \mathrm{BrN}_{3} \mathrm{~S}$ & 258.13 & 91 & $209-210$ \\
\hline 3 & $4-\mathrm{Br}$ & $\mathrm{C}_{8} \mathrm{H}_{8} \mathrm{BrN}_{3} \mathrm{~S}$ & 258.13 & 88 & $197-198$ \\
\hline 4 & $3-\mathrm{Cl}$ & $\mathrm{C}_{8} \mathrm{H}_{8} \mathrm{ClN}_{3} \mathrm{~S}$ & 213.68 & 94 & $198-199$ \\
\hline 5 & $4-\mathrm{Cl}$ & $\mathrm{C}_{8} \mathrm{H}_{8} \mathrm{ClN}_{3} \mathrm{~S}$ & 213.68 & 92 & $195-196$ \\
\hline 6 & $4-\mathrm{F}$ & $\mathrm{C}_{8} \mathrm{H}_{8} \mathrm{FN}_{3} \mathrm{~S}$ & 197.23 & 87 & $182-183$ \\
\hline 7 & $4-\mathrm{OMe}$ & $\mathrm{C}_{9} \mathrm{H}_{11} \mathrm{~N}_{3} \mathrm{OS}$ & 209.26 & 84 & $169-170$ \\
\hline 8 & $4-\mathrm{Me}$ & $\mathrm{C}_{9} \mathrm{H}_{11} \mathrm{~N}_{3} \mathrm{~S}$ & 193.26 & 90 & $215-216$ \\
\hline 9 & $3-\mathrm{NO}$ & $\mathrm{C}_{8} \mathrm{H}_{9} \mathrm{~N}_{4} \mathrm{O}_{2} \mathrm{~S}$ & 224.23 & 95 & $223-224$ \\
\hline 10 & $4-\mathrm{NO}$ & $\mathrm{C}_{8} \mathrm{H}_{8} \mathrm{~N}_{4} \mathrm{O}_{2} \mathrm{~S}$ & 224.23 & 91 & \\
\hline
\end{tabular}

(E)-2-benzylidenehydrazinecarbothioamide (1): Yield: $89 \%$, m.p. $157-158^{\circ} \mathrm{C} . \mathrm{UV} \lambda_{\max }(\mathrm{nm})$ : 309.50.IR $\left(\mathrm{KBr}, \mathrm{cm}^{-1}\right): v=1643.35(\mathrm{CH}=\mathrm{N}), 999.13(\mathrm{~N}-\mathrm{N}), 3387.00(-\mathrm{NH}), 3248.00\left(-\mathrm{NH}_{2}\right) .{ }^{1} \mathrm{H}$ NMR (DMSO, ppm): $\delta=8.048(\mathrm{~S}, 1 \mathrm{H} . \mathrm{CH}=\mathrm{N}), 7.387-7.801(\mathrm{~m} .5 \underline{\mathrm{H}} \mathrm{Ar}-\mathrm{H}) 8.190(\mathrm{~S}, 1 \mathrm{H} .-\mathrm{NH}), 7.982$ $\left(\mathrm{S}, 2 \mathrm{H} .-\mathrm{NH}_{2}\right) .{ }^{13} \mathrm{C}$ NMR (DMSO, ppm): $\delta\left(\mathrm{C}_{1}\right)=142.29(\overline{\mathrm{CH}}=\mathrm{N}), 134.19\left(\mathrm{C}_{2}\right), 128.64\left(\mathrm{C}_{3}\right)$, $127.30\left(\mathrm{C}_{4}\right), 129.84\left(\mathrm{C}_{5}\right), 128.66\left(\mathrm{C}_{6}\right), 128.66\left(\mathrm{C}_{7}\right), 178.01(\mathrm{C}=\mathrm{S}), \mathrm{M} . \mathrm{F}=\mathrm{C}_{8} \mathrm{H}_{9} \mathrm{~N}_{3} \mathrm{~S}, \mathrm{M} . \mathrm{W}=(179.24)$.

(E)-2-(3-bromobenzylidene)hydrazinecarbothioamide (2): Yield: 91\%, m.p. 209-210 ${ }^{\circ} \mathrm{C}$. UV $\lambda_{\max }(\mathrm{nm}): 318.00 . \mathrm{IR}\left(\mathrm{KBr}, \mathrm{cm}^{-1}\right): v=1641.42(\mathrm{CH}=\mathrm{N}), 997.20(\mathrm{~N}-\mathrm{N}), 3385.07(-\mathrm{NH}), 3250.05(-$ $\left.\mathrm{NH}_{2}\right) .{ }^{1} \mathrm{H}$ NMR $(\mathrm{DMSO}, \mathrm{ppm}): \delta=8.117(\mathrm{~S}, 1 \mathrm{H} . \mathrm{CH}=\mathrm{N}), 7.323-7.667(\mathrm{~m}, 4 \mathrm{H}, \mathrm{Ar}-\mathrm{H}), 8.171(\mathrm{~S}, 1 \mathrm{H} .-$ $\mathrm{NH}), 8.567\left(\mathrm{~S}, 2 \mathrm{H} .-\mathrm{NH}_{2}\right) .{ }^{13} \mathrm{C}$ NMR (DMSO, ppm): $\delta\left(\mathrm{C}_{1}\right)=140.75(\mathrm{CH}=\mathrm{N}), 136.48\left(\mathrm{C}_{2}\right), 126.84$ $\left(\mathrm{C}_{3}\right), 128.83\left(\mathrm{C}_{4}\right), \quad 132.34\left(\mathrm{C}_{5}\right), 122.25\left(\mathrm{C}_{6}\right), \quad 130.74\left(\mathrm{C}_{7}\right), 178.02(\mathrm{C}=\mathrm{S}), \quad \mathrm{M} . \mathrm{F}=\mathrm{C}_{8} \mathrm{H}_{8} \mathrm{BrN}_{3} \mathrm{~S}$. $\mathrm{M} / \mathrm{W}=(258.13)$.

(E)-2-(4-bromobenzylidene)hydrazinecarbothioamide (3): Yield: 88\%, m.p. 197-198 ${ }^{\circ}$. UV $\lambda_{\max }(\mathrm{nm}): 317.50$. IR $\left(\mathrm{KBr}, \mathrm{cm}^{-1}\right): v=1641.42(\mathrm{CH}=\mathrm{N}), 997.20(\mathrm{~N}-\mathrm{N}), 3385.00(-\mathrm{NH}), 3251.00(-$ $\mathrm{NH}_{2}$ ). ${ }^{1} \mathrm{H}$ NMR (DMSO, ppm): $\delta=8.015(\mathrm{~S}, 1 \mathrm{H} . \mathrm{CH}=\mathrm{N}), 7.283-7.864(\mathrm{~m}, 4 \mathrm{H} \mathrm{Ar}-\mathrm{H}), 8.239(\mathrm{~S}, 1 \mathrm{H} .-$ $\mathrm{NH}), 7.946\left(\mathrm{~S}, 2 \mathrm{H} .-\mathrm{NH}_{2}\right) .{ }^{13} \mathrm{C} \mathrm{NMR}(\mathrm{DMSO}, \mathrm{ppm}): \delta\left(\mathrm{C}_{1}\right)=140.23(\mathrm{CH}=\mathrm{N}), 132.23\left(\mathrm{C}_{2}\right), 128.50$ $\left(\mathrm{C}_{3}, \mathrm{C}_{7}\right) 132.23\left(\mathrm{C}_{4}, \mathrm{C}_{5}, \mathrm{C}_{6}\right), 172.58(\mathrm{C}=\mathrm{S}) . \mathrm{M} / \mathrm{F}=\mathrm{C}_{8} \mathrm{H}_{8} \mathrm{BrN}_{3} \mathrm{~S} . \mathrm{M} / \mathrm{W}=(258.13)$

(E)-2-(3--chlorobenzylidene)hydrazinecarbothioamide (4): Yield: 94\%, m.p. 198-199 ${ }^{\circ}$. UV $\lambda_{\max }(\mathrm{nm}): 315.00$. IR $\left(\mathrm{KBr}, \mathrm{cm}^{-1}\right): \quad v=1685.79(\mathrm{CH}=\mathrm{N}), 1078.21(\mathrm{~N}-\mathrm{N}), 3388.93(-\mathrm{NH}), 3230.77$ $\left(-\mathrm{NH}_{2}\right) .{ }^{1} \mathrm{H}$ NMR $(\mathrm{DMSO}, \mathrm{ppm}): \delta=8.041(\mathrm{~S}, 1 \mathrm{H} . \mathrm{CH}=\mathrm{N}), 7.403-7.654(\mathrm{~m}, 4 \mathrm{H} \mathrm{Ar}-\mathrm{H}), 8.181$ (S, 1H.-NH), $8.246\left(\mathrm{~S}, 2 \mathrm{H} .-\mathrm{NH}_{2}\right) .{ }^{13} \mathrm{C} \mathrm{NMR}$ (DMSO, ppm): $\delta\left(\mathrm{C}_{1}\right)=140.55(\mathrm{CH}=\mathrm{N}), 136.49\left(\mathrm{C}_{2}\right)$, $129.41\left(\mathrm{C}_{3}\right), 126.62\left(\mathrm{C}_{4}, \mathrm{C}_{7}\right), 130.46\left(\mathrm{C}_{5}\right), 133.78\left(\mathrm{C}_{6}\right), 178.21(\mathrm{C}=\mathrm{S}) . \mathrm{M} / \mathrm{F}=\mathrm{C}_{8} \mathrm{H}_{8} \mathrm{ClN}_{3} \mathrm{~S}$. $\mathrm{M} / \mathrm{W}=(213.68)$.

(E)-2-(4--chlorobenzylidene)hydrazinecarbothioamide (5): Yield: 92\%, m.p. 210-211 ${ }^{\circ} \mathrm{C}$. UV $\lambda_{\max }(\mathrm{nm}): 316.50$. IR $\left(\mathrm{KBr}, \mathrm{cm}^{-1}\right): v=1689.64(\mathrm{CH}=\mathrm{N}), 1078.21(\mathrm{~N}-\mathrm{N}), 3388.93(-\mathrm{NH}), 3371$ $\left(-\mathrm{NH}_{2}\right) .{ }^{1} \mathrm{H}$ NMR $(\mathrm{DMSO}, \mathrm{ppm}): \delta=8.060(\mathrm{~S}, 1 \mathrm{H} . \mathrm{CH}=\mathrm{N}), 7.439-7.842(\mathrm{~m}, 4 \mathrm{H} \mathrm{Ar}-\mathrm{H}), 8.021(\mathrm{~S}$, 1H.-NH), $8.222\left(\mathrm{~S}, 2 \mathrm{H} .-\mathrm{NH}_{2}\right) \cdot{ }^{13} \mathrm{C}$ NMR (DMSO, pm): $\delta\left(\mathrm{C}_{1}\right)=140.90(\mathrm{CH}=\mathrm{N}), 133.21\left(\mathrm{C}_{2}\right)$, $128.97\left(\mathrm{C}_{3}, \mathrm{C}_{7}\right), 128.72\left(\mathrm{C}_{4}, \mathrm{C}_{6}\right), 134.35\left(\mathrm{C}_{5}\right), 178.10(\mathrm{C}=\mathrm{S}) . \mathrm{M} / \mathrm{F}=\mathrm{C}_{8} \mathrm{H}_{8} \mathrm{ClN}_{3} \mathrm{~S} . \mathrm{M} / \mathrm{W}=(213.68)$. 
(E)-2-(4--Fulorobenzylidene)hydrazinecarbothioamide (6): Yield: 87\%, m.p. 195-196 ${ }^{\circ} \mathrm{C}$. UV $\lambda_{\max }(\mathrm{nm}): 311.00 . \mathrm{IR}\left(\mathrm{KBr}, \mathrm{cm}^{-1}\right): \quad v=1638.43(\mathrm{CH}=\mathrm{N}), 1093.64(\mathrm{~N}-\mathrm{N}), 3388.33(-\mathrm{NH}), 3238.48$ $\left(-\mathrm{NH}_{2}\right) .{ }^{1} \mathrm{H}$ NMR $(\mathrm{DMSO}, \mathrm{ppm}): \delta=8.051(\mathrm{~S}, 1 \mathrm{H} . \mathrm{CH}=\mathrm{N}), 7.367-7.819(\mathrm{~m}, 4 \mathrm{H} \mathrm{Ar}-\mathrm{H}), 7.948(\mathrm{~S}$, 1H.-NH), $8.356\left(\mathrm{~S}, 2 \mathrm{H} .-\mathrm{NH}_{2}\right) .{ }^{13} \mathrm{C}$ NMR (DMSO, ppm): $\delta\left(\mathrm{C}_{1}\right)=140.39(\mathrm{CH}=\mathrm{N}), 129.30\left(\mathrm{C}_{2}\right)$, $133.80\left(\mathrm{C}_{3}\right), 116.20\left(\mathrm{C}_{4}\right), 163.33\left(\mathrm{C}_{5}\right), 115.60\left(\mathrm{C}_{6}\right), 133.70\left(\mathrm{C}_{7}\right) 178.43(\mathrm{C}=\mathrm{S}) . \mathrm{M} / \mathrm{F}=\mathrm{C}_{8} \mathrm{H}_{8} \mathrm{FN}_{3} \mathrm{~S} . \mathrm{M} / \mathrm{W}$ $=(197.23)$.

(E)-2-(4--Methoxybenzylidene)hydrazinecarbothioamide (7): Yield:84\%, m.p. $182-183^{\circ} \mathrm{C} . U \mathrm{~V}$ $\lambda_{\max }(\mathrm{nm}): 320.50$. IR $\left(\mathrm{KBr}, \mathrm{cm}^{-1}\right): v=1625.99(\mathrm{CH}=\mathrm{N}), 1087.85(\mathrm{~N}-\mathrm{N}), 3381.21(-\mathrm{NH}), 3221.12(-$ $\left.\mathrm{NH}_{2}\right) .{ }^{1} \mathrm{H}$ NMR $(\mathrm{DMSO}, \mathrm{ppm}): \delta=8.101(\mathrm{~S}, 1 \mathrm{H} . \mathrm{CH}=\mathrm{N}), 7.712-7.741(\mathrm{~m}, 4 \mathrm{H} \mathrm{Ar}-\mathrm{H}) 3.786\left(\mathrm{OCH}_{3}\right)$, ,7.906 (S,1H.-NH), $7.990\left(\mathrm{~S}, 2 \mathrm{H} .-\mathrm{NH}_{2}\right) .{ }^{13} \mathrm{C} \mathrm{NMR}$ (DMSO, ppm): $\delta\left(\mathrm{C}_{1}\right)=142.36(\mathrm{CH}=\mathrm{N})$, 126.78( $\left(\mathrm{C}_{2}\right), 128.93\left(\mathrm{C}_{3}, \mathrm{C}_{7}\right), 114.18\left(\mathrm{C}_{4}, \mathrm{C} 6\right), 160.70\left(\mathrm{C}_{5}\right), 55.30\left(\mathrm{OCH}_{3}\right), 177.62(\mathrm{C}=\mathrm{S}) . \mathrm{M} / \mathrm{F}=$ $\mathrm{C}_{9} \mathrm{H}_{11} \mathrm{~N}_{3} \mathrm{OS} . \mathrm{M} / \mathrm{W}=(209.26)$.

(E)-2-(4--Methylbenzylidene)hydrazinecarbothioamide (8): Yield: 90\%, m.p. 169-170 ${ }^{\circ} \mathrm{C}$. UV $\lambda_{\max }(\mathrm{nm}): 313.50 \mathrm{IR}\left(\mathrm{KBr}, \mathrm{cm}^{-1}\right): v=1698(\mathrm{CH}=\mathrm{N}), 1093.43 \quad(\mathrm{~N}-\mathrm{N}), 3368.43(-\mathrm{NH}), 3258.84$ $\left(-\mathrm{NH}_{2}\right) .{ }^{1} \mathrm{H}$ NMR $(\mathrm{DMSO}, \mathrm{ppm}): \delta=8.150(\mathrm{~S}, 1 \mathrm{H} . \mathrm{CH}=\mathrm{N}), 7.197-7.683(\mathrm{~m}, 4 \mathrm{H} \mathrm{Ar}-\mathrm{H}) 2.491\left(\mathrm{CH}_{3}\right)$, 7.937 (S, 1H.-NH) 8.011(S 2H-NH $).{ }^{13} \mathrm{C}$ NMR (DMSO, ppm): $\delta\left(\mathrm{C}_{1}\right)=142.23(\mathrm{CH}=\mathrm{N}), 131.47$ $\left(\mathrm{C}_{2}\right), 127.30\left(\mathrm{C}_{3}, \mathrm{C}_{7}\right), 129.31\left(\mathrm{C}_{4}, \mathrm{C}_{6}\right), 139.67\left(\mathrm{C}_{5}\right), 21.07\left(\mathrm{CH}_{3}\right), 177.83(\mathrm{C}=\mathrm{S}), \mathrm{M} / \mathrm{F}=\mathrm{C}_{9} \mathrm{H}_{11} \mathrm{~N}_{3} \mathrm{~S}$. $\mathrm{M} / \mathrm{W}=(193.26)$.

(E)-2-(3--Nitrobenzylidene)hydrazinecarbothioamide (9): Yield: 95\%, m.p. 215-216 ${ }^{\circ} \mathrm{C}$ . UV $\lambda_{\max }(\mathrm{nm}): 311.50$. IR $\left(\mathrm{KBr}, \mathrm{cm}^{-1}\right): v=1643.35(\mathrm{CH}=\mathrm{N}), 1099.43(\mathrm{~N}-\mathrm{N}), 3373.50(-\mathrm{NH})$, $3265.49\left(-\mathrm{NH}_{2}\right){ }^{1} \mathrm{H}$ NMR (DMSO, ppm): $\delta=8.041(\mathrm{~S}, 1 \mathrm{H} . \mathrm{CH}=\mathrm{N}), 7.203-7.874(\mathrm{~m}, 4 \mathrm{H}, \mathrm{Ar}-\mathrm{H}), 8.032$ $(\mathrm{S}, 1 \mathrm{H} .-\mathrm{NH}), 8.185\left(\mathrm{~S}, 2 \mathrm{H} .-\mathrm{NH}_{2}\right) .{ }^{13} \mathrm{C} \mathrm{NMR}(\mathrm{DMSO}, \mathrm{ppm}): \delta\left(\mathrm{C}_{1}\right)=140.03(\mathrm{CH}=\mathrm{N}), 139.99\left(\mathrm{C}_{2}\right.$ , $\left.\mathrm{C}_{3}\right), 130.18\left(\mathrm{C}_{4}\right), 123.99\left(\mathrm{C}_{5}\right), 148.44\left(\mathrm{C}_{6}\right), 121.47\left(\mathrm{C}_{7}\right) 178.38(\mathrm{C}=\mathrm{S}) . \mathrm{M} / \mathrm{F}=\mathrm{C}_{8} \mathrm{H}_{9} \mathrm{~N}_{4} \mathrm{O}_{2} \mathrm{~S} . \mathrm{M} / \mathrm{W}=$ (224.23).

(E)-2-(4--Nitrobenzylidene)hydrazinecarbothioamide (10): Yield: 91\%, m.p. 223-224 ${ }^{\circ} \mathrm{C} . U \mathrm{~V}$ $\lambda_{\max }(\mathrm{nm}): 322.00$. IR $\left(\mathrm{KBr}, \mathrm{cm}^{-1}\right): v=1641.42(\mathrm{CH}=\mathrm{N}), 1099.43(\mathrm{~N}-\mathrm{N}), 3387.50(-\mathrm{NH}), 3256.94(-$ $\left.\mathrm{NH}_{2}\right) .{ }^{1} \mathrm{H}$ NMR $(\mathrm{DMSO}, \mathrm{ppm}): \delta=8.211(\mathrm{~S}, 1 \mathrm{H} . \mathrm{CH}=\mathrm{N}), 7.183-7.971 \quad(\mathrm{~m}, 4 \mathrm{H} \mathrm{Ar}-\mathrm{H}), 8.119(\mathrm{~S}$, 1H.-NH), $8.242\left(\mathrm{~S}, 2 \mathrm{H} .-\mathrm{NH}_{2}\right) .{ }^{13} \mathrm{C}$ NMR (DMSO, ppm): $\delta\left(\mathrm{C}_{1}\right)=140.77(\mathrm{CH}=\mathrm{N}), 139.63\left(\mathrm{C}_{2}\right)$, $123.84\left(\mathrm{C}_{3}, \mathrm{C}_{4}, \mathrm{C}_{6} \mathrm{C} 7\right), 151.76\left(\mathrm{C}_{5}\right), 178.42(\mathrm{C}=\mathrm{S}) . \mathrm{M} / \mathrm{F}=\mathrm{C}_{8} \mathrm{H}_{9} \mathrm{~N}_{4} \mathrm{O}_{2} \mathrm{~S} . \mathrm{M} / \mathrm{W}=(224.23)$.

Table 2. The ultraviolet absorption maxima $\left(\lambda_{\max }, \mathrm{nm}\right)$, infrared absorptions $\left(v, \mathrm{~cm}^{-1}\right)$ and NMR chemical shifts $(\delta, \mathrm{ppm})$ of substituted $(E)$-2-benzylidenehydrazinecarbothioamides

\begin{tabular}{|c|c|r|r|r|r|}
\hline ENTRY & $\mathrm{X}$ & $\mathrm{UV}\left(\lambda_{\max }\right) \mathrm{nm}$ & $\begin{array}{c}\mathrm{IR} v \mathrm{~cm}^{-1} \\
(\mathrm{CH}=\mathrm{N})\end{array}$ & ${ }^{1} \mathrm{H} \mathrm{CH}=\mathrm{N}(\mathrm{ppm})$ & $\begin{array}{c}{ }^{13} \mathrm{C} \\
\mathrm{C}=\mathrm{N}(\mathrm{ppm})\end{array}$ \\
\hline 1 & $\mathrm{H}$ & 309.50 & 1643.35 & 8.048 & 142.29 \\
\hline 2 & $3-\mathrm{Br}$ & 318.00 & 1641.42 & 8.117 & 140.75 \\
\hline 3 & $4-\mathrm{Br}$ & 317.50 & 1641.42 & 8.015 & 140.23 \\
\hline 4 & $3-\mathrm{Cl}$ & 315.00 & 1685.79 & 8.041 & 140.55 \\
\hline 5 & $4-\mathrm{Cl}$ & 316.50 & 1689.64 & 8.060 & 140.90 \\
\hline 6 & $4-\mathrm{F}$ & 311.00 & 1638.43 & 8.051 & 140.39 \\
\hline 7 & $4-\mathrm{OMe}$ & 320.50 & 1625.99 & 8.101 & 142.26 \\
\hline 8 & $4-\mathrm{Me}$ & 313.50 & 1698.00 & 8.150 & 142.23 \\
\hline 9 & $3-\mathrm{NO}_{2}$ & 311.50 & 1643.35 & 8.041 & 140.03 \\
\hline 10 & $4-\mathrm{NO}_{2}$ & 322.00 & 1641.42 & 8.211 & 140.77 \\
\hline
\end{tabular}




\section{RESULTS AND DISCUSSION}

\subsection{CHARACTERIZATION: UV SPECTRUM}

In the UV-visible spectra of the compound (fig-1) below $350 \mathrm{~nm}$, a single peak is observed for the of substituted $(E)$-2-benzylidenehydrazinecarbothioamides of (hydrazone) transition band due to $\pi-\pi^{*}$ at $309.00 \lambda_{\max }(\mathrm{nm})$. The observed peak is assigned to $\pi-\pi^{*}$ transition. According to the valence Band theory, as the conjugation increases, the energy difference between the highest occupied and the lowest unoccupied $\pi$-orbitals decreases and hence the wave length of the absorption band increases.

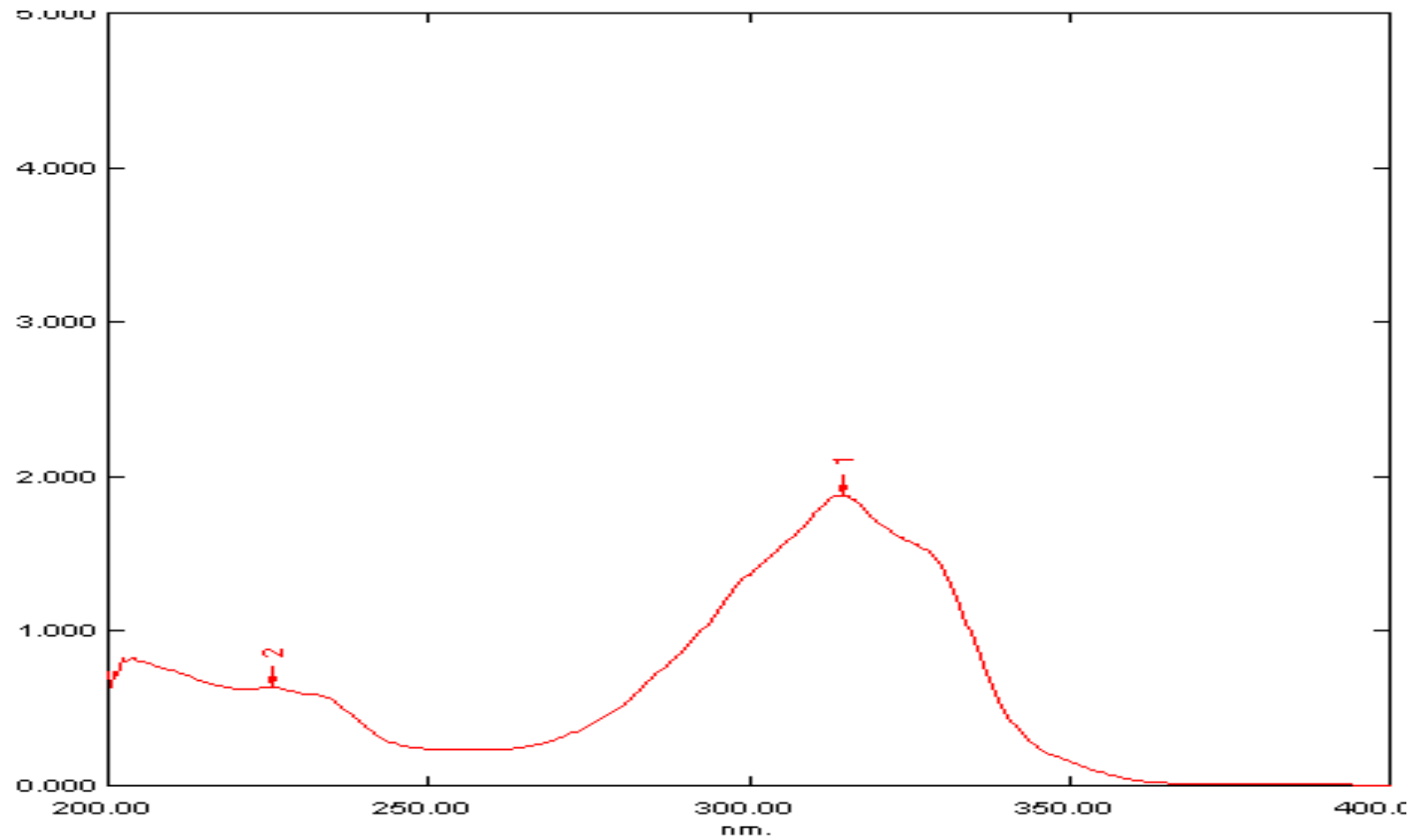

Fig:1 The UV spectrum of the (E)-2-benzylidenehydrazinecarbothioamide

\subsection{IR SPECTRUM}

The important IR frequencies (fig-2) of substituted (E)-2-benzylidenehydrazinecarbothioamides formed due to the condensation of thiosemaicarbazide with benzaldehyde are present in the table-2. A strong band is observed bending vibration for $(E)$-2-benzylidenehydrazinecarbothioamides of $(\mathrm{CH}=\mathrm{N})$ at around $1620-1700 \mathrm{~cm}^{-1}$ characteristic of the azomethine. The sharp peak at $3220 \mathrm{~cm}^{-1}$ corresponds to $\mathrm{N}-\mathrm{H}$ stretching and the broad absorption band at $3385 \mathrm{~cm}-1$ corresponds to the $\mathrm{NH}_{2}$ stretching. An absorption band was observed around the N-N stretching has been observed at 999 $\mathrm{cm}^{-1}$. 


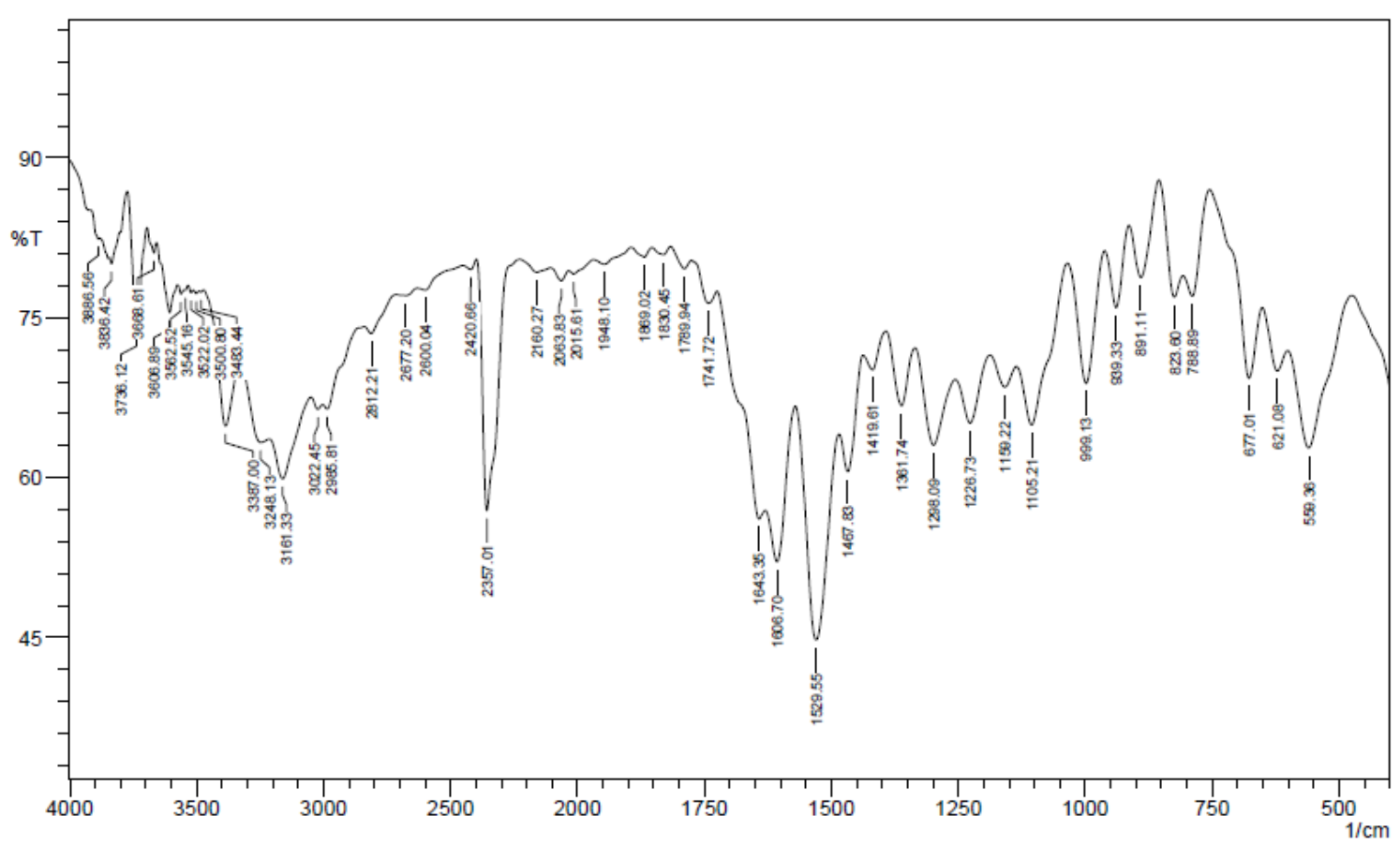

Fig: 2 The IR spectrum of $(E)$-2-benzylidenehydrazinecarbothioamide

\section{3. ${ }^{1}$ H NMR SPECTRUM}

The complete assignment of the ${ }^{\mathrm{I}} \mathrm{H}$ NMR spectra is given here (fig-3). The spectrum was recorded at $400 \mathrm{MHz}$. The assignment is done on the basis of chemical shifts, multiplicities and coupling constants. The 1H NMR spectrum (E)-2-benzylidenehydrazinecarbothioamide in DMSO$\mathrm{d}_{6}$ shows the one singlet for $\delta=8.048(\mathrm{~S}, 1 \mathrm{H} . \mathrm{CH}=\mathrm{N}),(\mathrm{ppm}), 8.190(\mathrm{~S}, 1 \mathrm{H} .-\mathrm{NH})$ and $7.982(\mathrm{~S}, 2 \mathrm{H}$.$\mathrm{NH}_{2}$ ) respectively. For this compound multiplet are observed at $7.387-7.801 \mathrm{ppm}$ which is assigned to protons ( 5 protons) of the aromatic rings. 


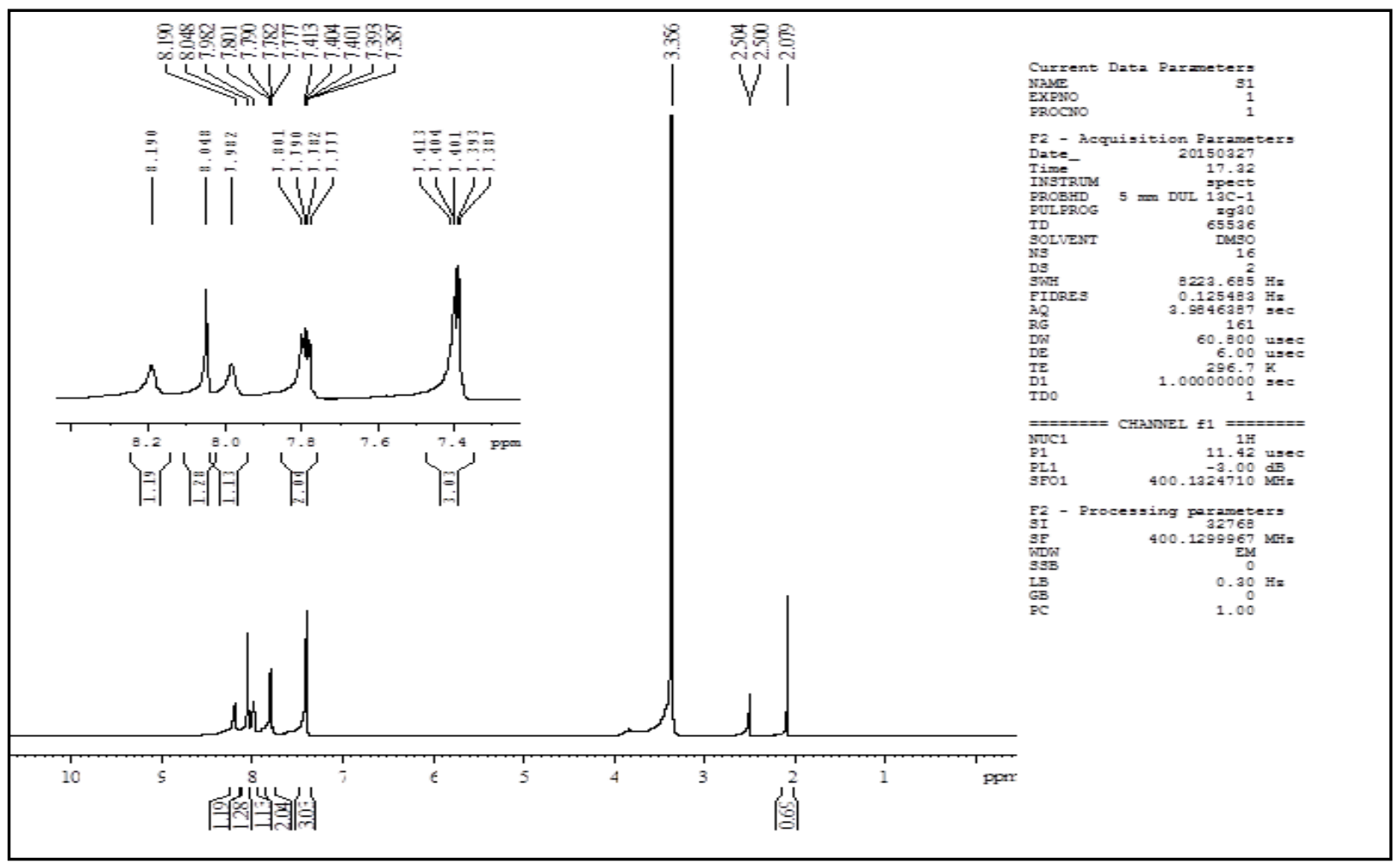

Fig: 3 The ${ }^{1} \mathrm{H}$ NMR spectrum of $(E)$-2-benzylidenehydrazinecarbothioamide

\section{Antimicrobial activities}

In an urge to develop new antimicrobial compound, a number of hydrazones were tested for their antimicrobial activities because of the evolution of drug-resistant microbial pathogens. The fast resistance of bacteria against antibiotics has become a widespread medical problem. Treatment options for these infections are often limited, especially in debilitated and immune compromised patients. The dramatically rising incidence of multi-drug resistant microbial infections in the past few decades has become a serious health care problem. The search for new antimicrobial agents will consequently always remain as an important and challenging task for medicinal chemists. The treatment of bacterial and fungal infectious diseases remains a challenging problem because of the increasing number of multi-drug microbial pathogens [39-41]. Nowadays, the design of new compounds able to deal with resistant bacteria, having new structures and new targets of action, has become one of the most important areas in the antibacterial research purpose [42].

\subsection{Antibacterial sensitivity assay}

Antibacterial sensitivity assay was performed using Kirby-Bauer [43] disc diffusion technique. In each Petri plate about $0.5 \mathrm{~mL}$ of the test bacterial sample was spread uniformly over the solidified Mueller Hinton agar using sterile glass spreader. Then the discs with $5 \mathrm{~mm}$ diameter made up of Whatmann No.1 filter paper, impregnated with the solution of the compound was placed on the medium using sterile forceps. The plates were incubated for 24 hours at $37{ }^{\circ} \mathrm{C}$ by keeping the plates upside down to prevent the collection of water droplets over the medium. After 24 hours, the plates were visually examined and the diameter values of the zone of inhibition were measured. Triplicate results were recorded by repeating the same procedure. The antibacterial screening effect of the synthesized substituted (E)-2-benzylidenehydrazinecarbothioamide compounds is shown in Fig. 4 Plates (1-10).

The antibacterial activities of all the synthesized substituted (E)-2-benzylidenehydrazinecarbothioamide compounds have been studied against three gram positive pathogenic strains Bacillus subtilis, Staphylococcus aureus, Streptococcus and two gram negative strains Escherichia coli and Pseudomonas aeruginosa species. The disc diffusion 
technique was followed using the Kirby-Bauer [43] method, at a concentration of $250 \mu \mathrm{g} / \mathrm{mL}$ with Ciprofloxacin used as the standard drug. The zone of inhibition is compared using Table $\mathbf{3}$ and the corresponding Clustered column Chart is shown in Fig. 5. The substituents parent $(\mathrm{H})$, 4- $\mathrm{Br}, 3-\mathrm{Cl}$, 4-Cl, 4-F, 4- $\mathrm{CH}_{3}, 3-\mathrm{NO}_{2}$ and 4- $\mathrm{NO}_{2}$ showed good activities against Bacillus subtilis species. All the substituents of $(E)$-2-benzylidenehydrazinecarbothioamides compounds showed good antibacterial activity against $S$. aureus. The only one $4-\mathrm{Cl}$ substituent has shown very good antibacterial activity against Streptococcus. The parent (H), 3-Br, 4-Br, 3-Cl, 4-F, 4- $\mathrm{CH}_{3}, 3-\mathrm{NO}_{2}$ and 4- $\mathrm{NO}_{2}$ substituents have shown good antibacterial activity. All the substituents of (E)-2benzylidenehydrazinecarbothioamides compounds showed good antibacterial activity against E.coli species. The parent $(\mathrm{H}), 3-\mathrm{Br}, 4-\mathrm{Br}, 3-\mathrm{Cl}, 4-\mathrm{Cl}, 4-\mathrm{F}, 4-\mathrm{CH}_{3}, 3-\mathrm{NO}_{2}$ and 4- $\mathrm{NO}_{2}$ substituents have shown good antibacterial activity against P.aeruginosa species. The only one $4-\mathrm{OCH}_{3}$ substituted compound has shown moderate antibacterial activity against P.aeruginosa species.

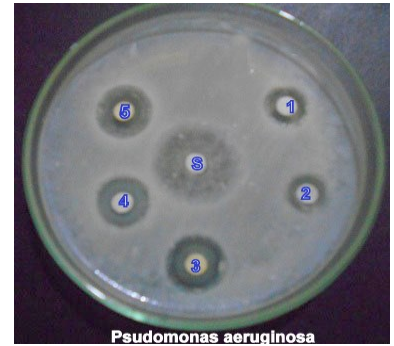

Plate-1

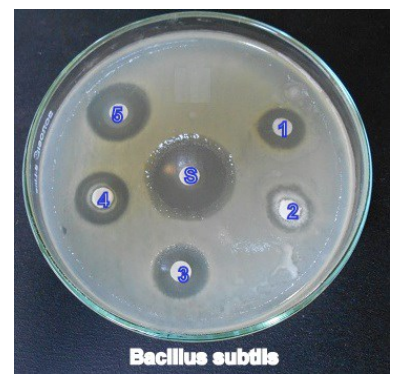

Plate-3

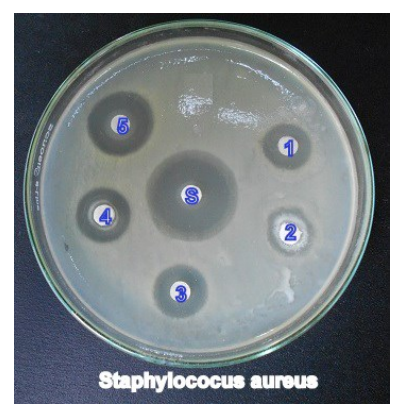

Plate-5

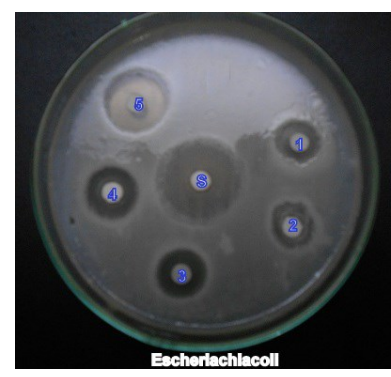

Plate-7

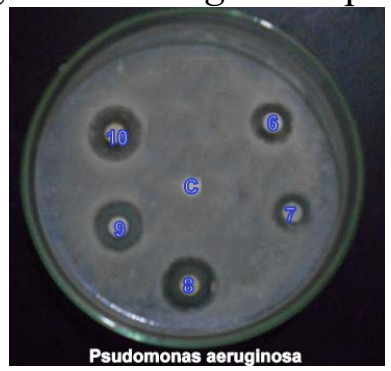

Plate-2

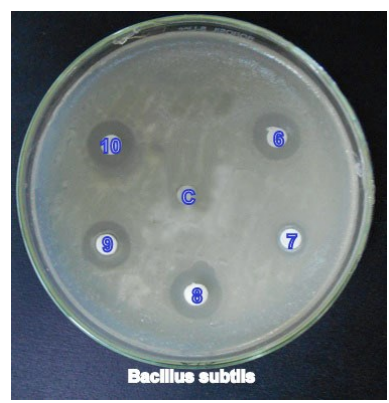

Plate-4

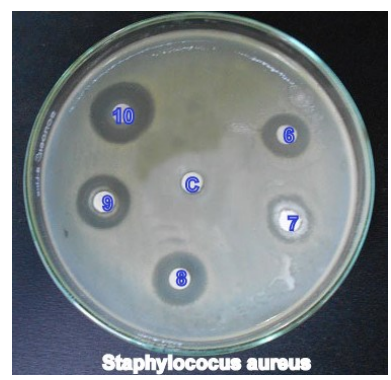

Plate-6

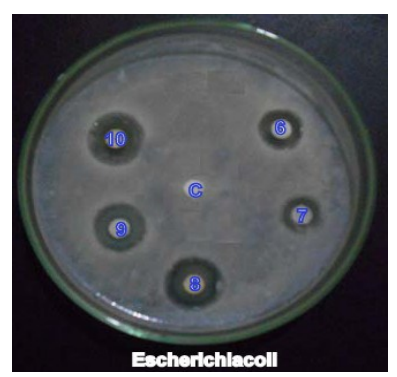

Plate-8 


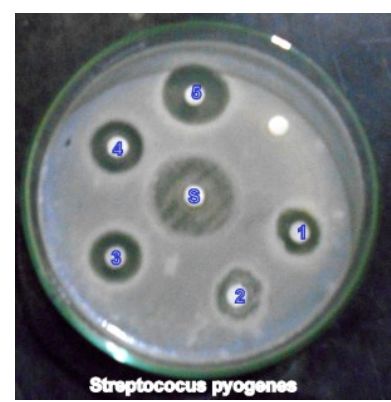

Plate-9

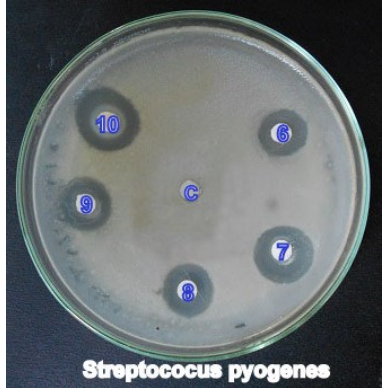

Plate-10

Fig. 4 Antibacterial activity of substituted (E)-2-benzylidenehydrazinecarbothioamide compounds

Table3. Antibacterial activity of zone of inhibition values of substituted

(E)-2-benzylidenehydrazinecarbothioamides

\begin{tabular}{|c|c|c|c|c|c|c|}
\hline \multirow{2}{*}{ S.No. } & \multirow{3}{*}{ Substituents } & \multicolumn{5}{|c|}{ Zone of inhibition(mm) } \\
\cline { 3 - 7 } & & \multicolumn{3}{|c|}{ Gram positive Bacteria } & \multicolumn{2}{c|}{ Gram negative Bacteria } \\
\cline { 3 - 7 } & & B.subtilis & $\begin{array}{l}\text { S. } \\
\text { aureus }\end{array}$ & Streptococcus & \multirow{2}{*}{ E.coli } & P.aeruginosa \\
\hline 1 & $\mathrm{H}$ & 10 & 10 & 12 & 10 & 12 \\
\hline 2 & $3-\mathrm{Br}$ & 8 & 9 & 10 & 12 & 10 \\
\hline 3 & $4-\mathrm{Br}$ & 12 & 13 & 13 & 13 & 14 \\
\hline 4 & $3-\mathrm{Cl}$ & 13 & 12 & 15 & 14 & 13 \\
\hline 5 & $4-\mathrm{Cl}$ & 15 & 14 & 18 & 16 & 15 \\
\hline 6 & $4-\mathrm{F}$ & 13 & 10 & 10 & 12 & 13 \\
\hline 7 & $4-\mathrm{OCH}$ & 8 & 9 & 8 & 15 & 6 \\
\hline 8 & $4-\mathrm{CH}_{3}$ & 14 & 12 & 12 & 12 & 10 \\
\hline 9 & $3-\mathrm{NO}_{2}$ & 12 & 11 & 11 & 11 & 11 \\
\hline 10 & $4-\mathrm{NO}_{2}$ & 15 & 13 & 14 & 15 & 13 \\
\hline Standard & Ciprofloxacin & 25 & 19 & 22 & 24 & 23 \\
\hline Control & DMSO & 0 & 0 & 0 & 0 & 0 \\
\hline
\end{tabular}

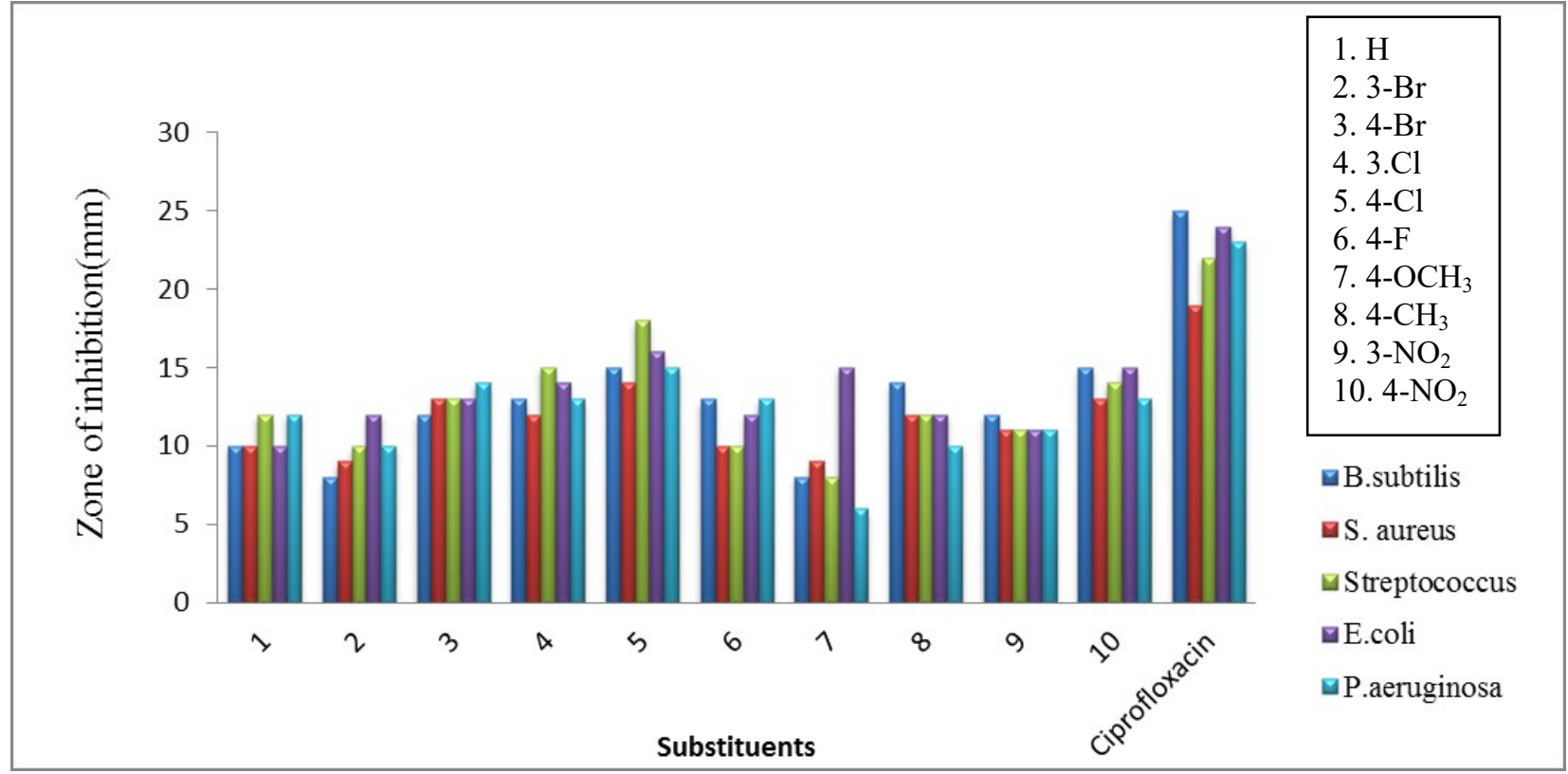

Fig. 5 Antibacterial activity of substituted $(E)$-2-benzylidenehydrazinecarbothioamide compounds Clustered column chart 


\subsection{Antifungal sensitivity assay}

Antifungal sensitivity assay was performed using Kirby-Bauer [43] disc diffusion technique. PDA medium was prepared and sterilized as above. It was poured (ear bearing heating condition) in the Petri-plate which was already filled with $1 \mathrm{ml}$ of the fungal species. The plate was rotated clockwise and counter clock-wise for uniform spreading of the species. The discs were impregnated with the test solution.

The test solution was prepared by dissolving $15 \mathrm{mg}$ of the hydrazone in $1 \mathrm{ml}$ of DMSO solvent. The medium was allowed to solidify and kept for 24 hours. Then the plates were visually examined and the diameter values of zone of inhibition were measured. Triplicate results were recorded by repeating the same procedure.

Table4. Antifungal activity of Zone of inhibition values of substituted (E)-2-benzylidenehydrazinecarbothioamides

\begin{tabular}{|c|c|c|c|c|}
\hline \multirow{2}{*}{ S. No. } & \multirow{2}{*}{ Substituents } & \multicolumn{3}{|c|}{ Zone of inhibition(mm) } \\
\cline { 3 - 5 } & & A. flavus & A. niger & T. viride \\
\hline 1 & $\mathrm{H}$ & 8 & 11 & 9 \\
\hline 2 & $3-\mathrm{Br}$ & 10 & 10 & 12 \\
\hline 3 & $4-\mathrm{Br}$ & 12 & 8 & 10 \\
\hline 4 & $3-\mathrm{Cl}$ & 8 & 9 & 9 \\
\hline 5 & $4-\mathrm{Cl}$ & 8 & 15 & 12 \\
\hline 6 & $4-\mathrm{F}$ & 10 & 8 & 9 \\
\hline 7 & $4-\mathrm{OCH}_{3}$ & 11 & 10 & 12 \\
\hline 8 & $4-\mathrm{CH}_{3}$ & 10 & 8 & 9 \\
\hline 9 & $3-\mathrm{NO}_{2}$ & 8 & 8 & 4 \\
\hline 10 & $4-\mathrm{NO}_{2}$ & 9 & 10 & - \\
\hline Standard & Ciprofloxacin & 16 & 16 & - \\
\hline Control & DMSO $^{2}$ & - & & 17 \\
\hline
\end{tabular}




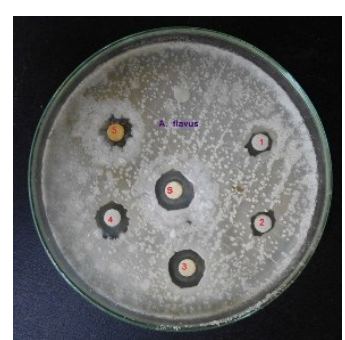

Plate-1

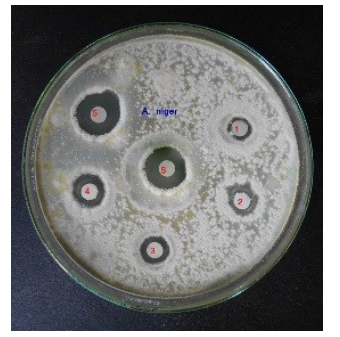

Plate-3

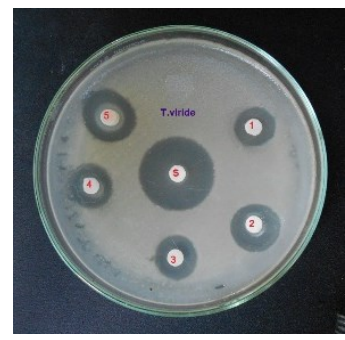

Plate-5

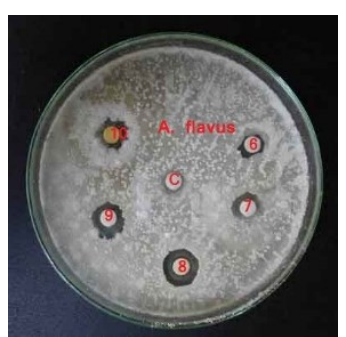

Plate-2

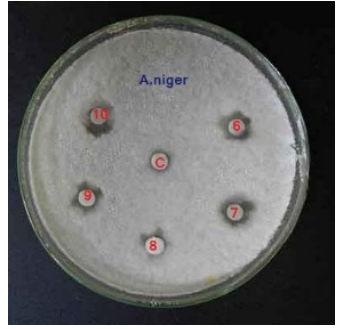

Plate-4

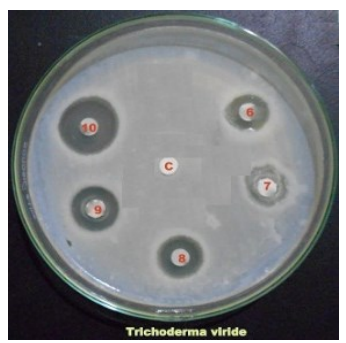

Plate-6

Fig. 6 Antifungal activity of substituted $(E)$-2-benzylidenehydrazinecarbothioamide compounds

Antifungal assay has been performed using Kirby-Bauer [43] disc diffusion technique. The antifungal activities of the entire substituted $(E)$-2-benzylidenehydrazinecarbothioamide have been studied and are shown in Fig. 6 for Plates (1-4). The zone of inhibition values of the antifungal activities is given in Table 5. The clustered column chart was shown in Fig. 7 and it reveals that the $4-\mathrm{Br}$ and $4-\mathrm{OCH}_{3}$ compounds have shown good activity against $A$. flavus. The $3-\mathrm{Br}$ and $4-\mathrm{Cl}$ substituted compounds have shown excellent activity against A.niger. Also, 3-Br, 4-Br, 4-Cl, 4-F and $4-\mathrm{CH}_{3}$ compounds have shown good activity against $T$.viride. The remaining compounds have shown moderate activity against all the fungi.

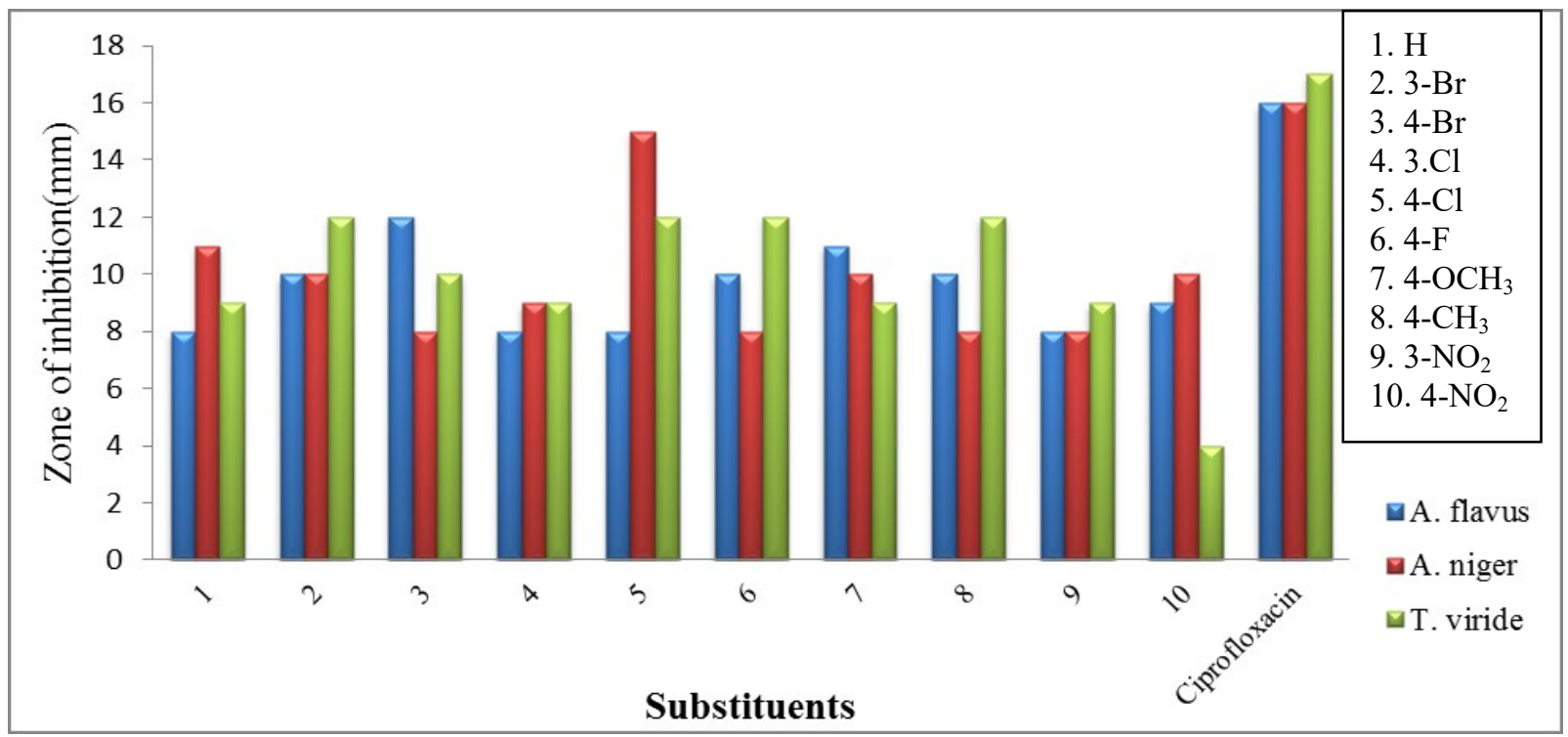

Fig. 7 Antifungal activity of substituted (E)-2-benzylidenehydrazinecarbothioamide compounds (Clustered column chart) 


\section{CONCLUSIONS}

The authors have been synthesised some substituted $(E)$-2-benzylidenehydrazinecarbothioamide compounds using condensation method. The antimicrobial activities of all the synthesized hydrazone compounds have been evaluated using Bauer-Kirby disc diffusion technique. Most of the synthesized compounds found to shown moderate antibacterial as well as antifungal activity. The 4$\mathrm{Cl}$ substituted compound has shown good activity against Spectrococcus and A. niger species.

\section{REFERENCE}

[1] French F.A., Blanz E.J., Journal of medicinal Chemistry. 9 (1966) 585-589

[2] Idem, Cancer Research., 26 (1966) 1638.

[3] Idem, Ibid, 28 (1968) 2419.

[4] Agrawal K.C., Booth A.B., Sartorelli AC., Journal of medicinal Chemistry. 11 (1968) 700.

[5] M.Mohan, N.S.Gupta, M.P.Gupta, A Kurnar, M.Kurnar, N.K.Jha, Inorganica.Chimica Acta., 152 (1988) 25-36

[6] Mohan M., Kurnar A., Kurnar M., Jha N.K., Ibid, 136 (1987) 65.

[7] Ma T.S., Tien T.M., Antibiotics and Chemotherapy. 3 (1953) 491.

[8] Abbert Q.,Nature. 9 (1961) 370.

[9] Price IM., Federation Proceedings., 20 (1961) 223.

[10] Sacconi L., Journal of the American Chemical Society ., 74 (1952) 4503

[11] Korolkovas A., Burckhalter IH., Essentials of Medicinal Chemistry, Interscience Publication. N.Y(1976).

[12] Loncle C., Brunel J.M., Vidal N., Dherbomez M., Letourneux Y., European journal of Medicinal Chemistry 39 (2004) 1067-1071.

[13] Papakonstantinou-Garoufalias S., Pouli N., Marakos P., Chytyroglou-Ladas A., Farmaco. 57 (2002) 973-977.

[14] B. K. Kaymakcioglu and S. Rollas, Farmaco, 2002, 57, 595-599.

[15] Maccari R., Ottana R., Vigorita M.G., Bioorganic Medicinal Chemistry Letters. 15 (2005) 2509-2513.

[16] Cocco M.T., Congiu C., Onnis V., Pusceddu M.C., Schivo M.L., De Logu A., European Journal of Medicinal Chemistry. 34 (1999) 1071-1076.

[17] Rando D.G., Sato D.N., Siqueira L., Malvezzi A., Leite C. Q. F., de Amaral A. T., Ferreira E. I., Tavares L.C., Bioorgani and Medicinal Chemistry 10 (2002)557-560.

[18] Patole J., Shingnapurkar D., Padhye S., Ratledge C., Bioorganic Medicinal Chemistry Letters 6 (2006) 1514-1517.

[19] Maiti A., Ghosh S., Journal of Inorganic Biochemistry 36 (1989) 131-139.

[20] Badiger D.S., Hunoor R.S., Patil B.R., Vadavi R.S., Mangannavar C.V., Muchchandi I.S., Patil Y.P., Nethaji M., Gudasi K.B., Inorganica Chimica Acta. 384 (2012) 197-203.

[21] Rollas S., Kucukguzel S. G., Molecules, 12 (2007) 1910-1939.

[22] Sreeja P. B., Kurup M. R. P., Kishore A., Jasmin C., Polyhedron. 23 (2004) 575-581.

[23] Patai S., Interscience, NewYork, (1970) 149-180.

[24] Seleem HS., El-Inany GA., El-Shetary BA., Mousa MA., Chemistry Central Journal. 5 (2011) 1186 
[25] R. Narang, B. Narasimhan, S. Sharma, Current Medicinal Chemistry 19 (2012) 569-612.

[26] Terzio_glu N., Gürsoy A., European Journal of Medicinal Chemistry. 38 (2003) 781-786.

[27] Vicini P., Incerti M., Doytchinova I., La Colla P., Busonera B., Loddo R., European Journal of Medicinal Chemistry 41 (2006) 624-632.

[28] Gürsoy A., Terzioglu N., Otuk G., European Journal of Medicinal Chemistry 32 (1997) 753-757.

[29] Ulusoy N., Gürsoy A., Otuk G., Il Farmaco 56 (2001) 947-952.

[30] Rochlitz C.F., Damon L.E., Russi M.B., Geddes A., Cadman E.C., Cancer Chemother. Pharmacol. 21 (1988) 319-322.

[31] Procopio G., Guadalupi V., Giganti M.O., Mariani L., Salvioni R., Nicolai N., Capone F., Valdagni R., Bajetta E., BJU International. 108 (2010) 223-228.

[32] Shi W., Nacev B.A., Aftab B.T., Head S., Rudin C.M., Liu J.O., Journal of Medicinal Chemistry 54 (2011) 7363-7374.

[33] Bhaskar V.H., Mohite P.B., Optoelectron J., Biomedical Materials. 2 (2010) 249-259.

[34] Galmarini C.M.,. Mackey J.R, Dumontet C., Leukemia. 15 (2001) 875-890.

[35] Ghannoum M.A., Louis B.R., Clin., Microbiologial Reviews. 12 (1999) 501-517.

[36] Kitaev Y.P., Buzykin B.I., Troepol'skaya T.V., Russ., Chemical Reviews. 39 (1970) 441456.

[37] Wu A.M., Senter P.D., Nature Biotechnology 23 (2005) 1137-1146.

[38] Nataliya P., Belskaya., Wim Dehaen., Vasiliy Bakuleva A., Arkivoc 1 (2010) 275-332

[39] Diana Camelia Nuţa., Carmen Balotescu Chifiriuc., Alexandru Vasile Missir., Ileana Cornelia Chiriţa., Carmellina Daniela Badiceanu., Farmacia. 58(1) ( 2010) 38-45.

[40] Omar K., Geronikaki A., Zoumpoulakis P., Camoutsis C., Sokovic M., Ciric A., Glamoclija., J., Bioorganic Medicinal Chemistry 18 (2010) 426-432.

[41] Ioana Moş., Otilia Micle., Mihaela Zdranca., Mariana Mureşan., Laura Vicaş., Farmacia, 58(5) (2010) 637-645

[42] Zani, F., Vicini, P., Incerti M., European Journal of Medicinal Chemistry., 39 (2004) 135140.

[43] Bauer A. W., Kirby W. M. M., Sherris J. C., Truck M., American Journal of Clinical. Pathology 45 (1966) 493-496. 